Pacific Journal of Mathematics

THE UNIFORMIZING FUNCTION FOR CERTAIN SIMPLY 


\title{
THE UNIFORMIZING FUNCTION FOR CERTAIN SIMPLY CONNECTED RIEMANN SURFACES
}

\author{
Howard B. Curtis, JR.
}

This paper contains a definition of a class of simply connected Riemann surfaces, the determination of the type of a surface from this class, and a representation of the uniformizing function and its derivative as infinite products of quotients as well as quotients of infinite products.

Definition of the class of surfaces. Let $\left\{a_{2 n-1}\right\}_{n=1}^{\infty}$ and $\left\{b_{n}\right\}_{n=1}^{\infty}$ be two sequences of real numbers such that for $n \geqq 1$,

$$
0<a_{2 n-1}<b_{2 n-1}<b_{2 n}
$$

and $b_{2 n+1}<b_{2 n}$. A surface $F$ of the class to be discussed consists of sheets $S_{n}, n=1,2,3, \cdots$, over the $w$-sphere, where for $S_{n}$ a copy of the $w$-sphere,

(a) $S_{1}$ is slit along the real axis from $a_{1}$ to $b_{1}$.

(b) For $n \geqq 1, S_{2 n}$ is slit along the real axis from $a_{2 n-1}$ to $b_{2 n-1}$ and from $b_{2 n}$ to $+\infty$.

(c) For $n \geqq 1, S_{2 n+1}$ is slit along the real axis from $a_{2 n+1}$ to $b_{2 n+1}$ and from $b_{2 n}$ to $+\infty$.

(d) For $n \geqq 1, S_{n}$ is joined to $S_{n+1}$ along the slits to make the $b_{n}$ coincide and to form first order branch points at the endpoints of the slits.

The uniformizing function. Because $F$ is simply connected and noncompact, there exists a unique function $g$ which maps $F$ schlichtly and conformally onto $\{|z|<R \leqq \infty\}$, where for $f(z)=g^{-1}(z), f(0)=$ $0 \in S_{1}$ and $f^{\prime}(0)=1$. Two surfaces of hyperbolic type are obtained by slitting each sheet of $F$ along the uncut parts of the real axis, and an application of the reflection principle to the uniformizing function of one of these surfaces shows that $f(z)$ is real for real $z$. Let $f\left(\alpha_{2 k-1}\right)=a_{2 k-1}, f\left(-\beta_{k}\right)=b_{k}, f\left(\gamma_{2 k}\right)=\infty \in S_{2 k}$ and $S_{2 k+1}, f\left(-\gamma_{1}\right)=\infty \in S_{1}$, and $f\left(\delta_{k}\right)=0 \in S_{k}$. The image of $F$ in the $z$-plane satisfies the following properties. The image of $S_{n}$ is a region which is symmetric about the real axis. $S_{1}$ is mapped onto a domain containing the origin and bounded by a simple closed curve $C_{1}$ which intersects the real axis at $-\beta_{1}$ and $\alpha_{1}$. For $n \geqq 2, S_{n}$ is mapped onto an annular region about the origin and bounded by two simple closed curves $C_{n-1}$ and $C_{n}$, which

Received July 24, 1964. Research for this paper was supported by a grant from the University of Texas Research Institute and revision of the paper was completed while the author was a Research Fellow in Mathematics at Rice University. 
are images of cuts. For $n$ odd, $C_{n}$ intersects the real axis at $-\beta_{n}$ and $\alpha_{n}$, while for $n$ even, $C_{n}$ intersects the real axis at $-\beta_{n}$ and $\gamma_{n}$. Furthermore, for $k \geqq 1$,

$$
-\beta_{k+1}<-\beta_{k}<-\gamma_{1}<0<\alpha_{2 k-1}<\delta_{2 k}<\gamma_{2 k}<\delta_{2 k+1}<\alpha_{2 k+1} .
$$

The approximating closed surfaces, Let $F_{n}$ be the surface formed from the first $2 n+2$ sheets of $F$ with the slit in $S_{2 n+2}$ from $b_{2 n+2}$ to $\infty$ deleted, so that $F_{n}$ is a compact, simply connected surface.

Notation. $\quad \alpha_{\varphi}^{*}=1-z / \alpha_{\varphi}, \quad \beta_{\varphi}^{*}=1+z / \beta_{\varphi}$,

$$
\gamma_{\varphi}^{*}=1-z / \gamma_{\varphi}, \quad \delta_{\varphi}^{*}=1-z / \delta_{\varphi} \text {. }
$$

LEMMA 1. Let $R_{n}$ be the unique rational function which maps the z-sphere one-to-one onto the simply connected compact surface $F_{n}$ with $R_{n}(0)=0 \in S_{1}, R_{n}^{\prime}(0)=1$, and $R_{n}(\infty)=\infty \in S_{2 n+2}$. Then

$$
R_{n}(z)=\left[z /\left(1+z / \gamma_{1, n}\right)\right]\left[\prod_{k=2}^{2 n+2} \delta_{k, n}^{*}\right] /\left[\prod_{k=1}^{n}\left(\gamma_{2 k, n}^{*}\right)^{2}\right]
$$

and

$$
R_{n}^{\prime}(z)=\left[1 /\left(1+z / \gamma_{1, n}\right)^{2}\right]\left[\prod_{k=0}^{n} \alpha_{2 k+1, n}^{*}\right]\left[\prod_{k=1}^{2 n+1} \beta_{k, n}^{*}\right] /\left[\prod_{k=1}^{n}\left(\gamma_{2 k, n}^{*}\right)^{3}\right] .
$$

Proof. The representations of $R_{n}$ and $R_{n}^{\prime}$ must contain factors shown and can contain no more. The $\alpha_{2 k+1, n},-\beta_{k, n}, \gamma_{2 k, n}$, and $\delta_{k, n}$, which are ordered in the same manner as the $\alpha_{2 k+1},-\beta_{k}, \gamma_{2 k}$, and $\delta_{k}$, are images of $a_{2 k+1}, b_{k}, \infty$, and 0 , respectively, under $R_{n}^{-1}$.

\section{LEMMA 2. $F$ is parabolic.}

Proof. Suppose that $F$ is hyperbolic, and thus $g$ maps $F$ onto $\{|z|<R<\infty\}$. If $D_{n}$ is the $z$-plane slit along the real axis from $-\beta_{2 n+1, n}$ to $-\infty$, then $\zeta=\psi_{n}(z)=g\left[R_{n}(z)\right]$ defines a Schlicht mapping of $D_{n}$ onto a simply connected region $\Delta_{n}$ of the $\zeta$-plane bounded by $C_{2 n+2}$ and the segment $\left(-\beta_{2 n+2},-\beta_{2 n+1}\right)$. If $T_{n}(z)=z\left(1-z / 4 \beta_{2 n+1, n}\right)^{-2}$, then $\zeta=\psi_{n}\left[T_{n}(z)\right]$ defines a properly normalized, Schlicht mapping of $\left\{|z|<4 \beta_{2 n+1, n}\right\}$ onto $\Delta_{n}$ such that if the Koebe Distortion Theorem is applied to this map, then $\beta_{2 n+1, n} \leqq d\left(0, C_{2 n+2}\right) \leqq R<\infty$, where $d\left(0, C_{2 n+2}\right)$ is the distance from $\zeta=0$ to the curve $C_{2 n+2}$. Thus there exists a subsequence $\left\{\beta_{2 n_{j}+1, n_{j}}\right\}$ such that $\beta_{2 n_{1}+1, n_{j}} \rightarrow A \leqq R$ as $j \rightarrow \infty$, and $\psi_{n \text {, }}$ is a Schlicht mapping of $D_{n j}$ onto $\Delta_{n j^{\circ}}$ If $D$ is the $z$-plane slit along the negative real axis from $-A$ to $-\infty$, then $\left\{\psi_{n g}\right\}$ forms a family of functions which is normal in $D$, and hence there exists a subsequence $\left\{\psi_{i}\right\}$ such that as $i \rightarrow \infty, \psi_{i}(z) \rightarrow \psi(z)$ uniformly on any compact sub- 
set of $D$. Because $D_{i} \rightarrow D$ and $\psi_{i}(z) \rightarrow \psi(z)$ as $i \rightarrow \infty$, then $\Delta_{i} \rightarrow$ $\{|z|<R\}$ and $\psi$ maps $D$ onto $\{|\zeta|<R\}$ in a one-to-one manner. ([1], p. 18). Then $R_{i}(z)=f\left[\psi_{i}(z)\right] \rightarrow f[\psi(z)]=H(z)$ uniformly on any compact subset of $D$ as $i \rightarrow \infty$, where $H$ is meromorphic in $D$, while $H(z) \not \equiv \infty$ because $R_{i}(0)=0$. $H$ maps $D$ onto $F$.

Now let $D^{*}$ be the $z$-plane slit along the real axis from $-A$ to $+\infty$. For $i$ sufficiently large, $R_{i}(z)$ assumes no negative real values in any compact subset of $D^{*}$, and thus $\left\{R_{i}\right\}$ is a family of functions which is normal in $D^{*}$. Therefore, there exists a subsequence $\left\{R_{m}\right\}$ of $\left\{R_{i}\right\}$ such that as $m \rightarrow \infty, R_{m}(z) \rightarrow G(z)$ uniformly on any compact subset of $D^{*} . \quad H$ and $G$ have a common domain of convergence, so that $G$ is the analytic continuation of $H$. Then $w=G(z)$ defines a mapping of the $z$-plane punched at $z=A$ and $\infty$ one-to-one and conformally onto an open doubly connected Riemann surface $F^{*}$ of which $F$ is a subsurface obtained by inserting some slits in $F^{*}$ over the real axis. This is impossible, as is clear from the definition of $F$. Hence $R=\infty$.

Lemma 3. $R_{n}(z) \rightarrow f(z)$ uniformly on any compact subset of the $z$-plane as $n \rightarrow \infty$.

Proof. Because $\Delta_{n} \rightarrow\{|\zeta|<\infty\}$ as $n \rightarrow \infty$, it follows ([1], p. 18) that $z=R_{n}^{-1}[f(\zeta)] \rightarrow \zeta=g\left[R_{n}(z)\right]$ uniformly on any compact subset of the $\zeta$-plane as $n \rightarrow \infty$. Also, $D_{n} \rightarrow\{|z|<\infty\}$ and $R_{n}(z) \rightarrow f(z)$ uniformly on any compact subset of the $z$-plane as $n \rightarrow \infty$.

LEMMA 4. $\alpha_{2 k-1, n} \rightarrow \alpha_{2 k-1}, \beta_{k, n} \rightarrow \beta_{k}, \gamma_{2 k, n} \rightarrow \gamma_{2 k}$, and $\delta_{k, n} \rightarrow \delta_{k}$ as $n \rightarrow \infty$ 。

Proof. This is a consequence of Hurwitz's Theorem.

LEMMA 5. The infinite product

$$
\pi(z)=\left[z /\left(1+z / \gamma_{1}\right)\right] \prod_{k=1}^{\infty}\left[\delta_{2 k}^{*} \delta_{2 k+1}^{*} /\left(\gamma_{2 k}^{*}\right)^{2}\right]
$$

converges uniformly on any compact subset of the z-plane.

Proof. Since $\gamma_{2 k} \rightarrow \infty$ and $\delta_{k} \rightarrow \infty$ as $k \rightarrow \infty$, then for any $R>0$, there exists $n_{0}=n_{0}(R)$ such that for $k \geqq n_{0}, \delta_{k}>R$ and $\gamma_{2 k}>R$. Then consider

$$
M_{p}(z)=\prod_{k=n_{0}}^{n_{0}+p}\left[\begin{array}{ll}
\delta_{2 k}^{*} & \left.\delta_{2 k+1}^{*} /\left(\gamma_{2 k}^{*}\right)^{2}\right]
\end{array}\right.
$$

$M_{p}$ is holomorphic for $|z| \leqq R$ and $M_{p}(z) \neq 0$ for $|z| \leqq R$. A sufficient 
condition for the uniform convergence of $M_{p}(z)$ in $E=\{|z| \leqq R\}$ as $p \rightarrow \infty$ is the uniform convergence in $E$ of

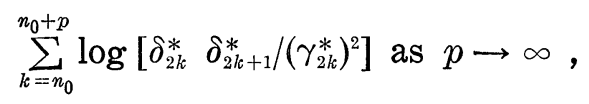

where each logarithm is the principal value. By the Cauchy criterion, this last sequence converges uniformly in $E$ provided for $z \in E$ and for any $\varepsilon>0$, there exists $N(\varepsilon)>0$ such that for $n>N(\varepsilon)$ and $p>0$,

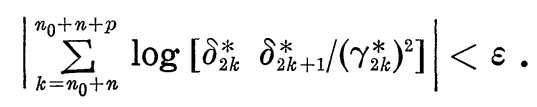

Now since $\delta_{2 k}<\delta_{2 k+1}$ and since $\gamma_{2 k}<\delta_{2 k+2}<\delta_{2 k+3}$, then for $m \geqq 1$ and $p>0$,

$$
0<\sum_{k=n_{0}+n}^{n_{0}+n+p}\left[1 /\left(\delta_{2 k}\right)^{m}+1 /\left(\delta_{2 k+1}\right)^{m}-2 /\left(\gamma_{2 k}\right)^{m}\right]<2 /\left(\delta_{2 n_{0}+2 n}\right)^{m} .
$$

Then for all $p>0$ and $z \in E$,

$$
\begin{aligned}
& \left|\sum_{k=n_{0}+n}^{n_{0}+n+p} \log \left[\delta_{2 k}^{*} \delta_{2 k+1}^{*} /\left(\gamma_{2 k}^{*}\right)^{2}\right]\right|=\left|-\sum_{m=1}^{\infty}\left[z^{m} / m\right] \sum_{k=n_{0}+n}^{n_{0}+n+p}\left[\left(1 / \delta_{2 k}^{m}\right)+\left(1 / \delta_{2 k+1}^{m}\right)-2 / \gamma_{2 k}^{m}\right]\right| \\
& \leqq \sum_{m=1}^{\infty}\left[R^{m} / m\right]\left[2 /\left(\delta_{2 n_{0}+2 n}\right)^{m}\right] \leqq 2 \sum_{m=1}^{\infty}\left[R /\left(\delta_{2 n_{0}+2 n}\right)\right]^{m}=2 R /\left(\delta_{2 n_{0}+2 n}-R\right) \text {. }
\end{aligned}
$$

Since $\delta_{2 n_{0}+2 n} \rightarrow \infty$ as $n \rightarrow \infty$, the Cauchy criterion is satisfied and $M_{p}$ converges uniformly in $E$. Thus $\Pi(z)$ converges uniformly in any compact subset of the $z$-plane.

LEMMA 6. $\pi(z)=f(z)$.

Proof. As a consequence of Lemma 4, there exists $r>0$ such that $R_{n}(z) / z \neq 0$ and $\pi(z) / z \neq 0$ for $|z|<r$, while each of these quotients defines a function which is holomorphic for $|z|<r$ and takes the value 1 at $z=0$. Thus using the principal value of the logarithm, for $|z|<r$,

$$
\begin{aligned}
\log \left[R_{n}(z) / z\right] & -\log [\pi(z) / z]=\log \left[R_{n}(z) / \pi(z)\right]=\log \left[\left(1+z / \gamma_{1}\right) /\left(1+z / \gamma_{1, n}\right)\right] \\
& -\sum_{m=1}^{\infty}\left\{z^{m} / m\right\}\left\{\sum_{k=1}^{n+1}\left(1 / \delta_{2 k, n}^{m}\right)+\sum_{k=1}^{n}\left(1 / \delta_{2 k+1, n}^{m}\right)-\sum_{k=1}^{n}\left(2 / \gamma_{2 k, n}^{m}\right)\right. \\
& \left.-\sum_{k=1}^{\infty}\left[\left(1 / \delta_{2 k}^{m}\right)+\left(1 / \delta_{2 k+1}^{m}\right)-2 / \gamma_{2 k}^{m}\right]\right\} .
\end{aligned}
$$

Therefore, for $n_{0}>2$, as $n \rightarrow \infty$,

$$
0 \leqq \lim \sup \mid \sum_{k=1}^{n+1}\left(1 / \delta_{2 k, n}^{m}\right)+\sum_{k=1}^{n}\left(1 / \delta_{2 k+1, n}^{m}\right)
$$




$$
\begin{aligned}
& -\sum_{k=1}^{n}\left(2 / \gamma_{2 k, n}^{m}\right)-\sum_{k=1}^{\infty}\left[\left(1 / \delta_{2 k}^{m}\right)+\left(1 / \delta_{2 k+1}^{m}\right)-2 / \gamma_{2 k}^{m}\right] \mid \\
& \leqq \lim \sup \mid \sum_{k=n_{0}}^{n+1}\left(1 / \delta_{2 k, n}^{m}\right)+\sum_{k=n_{0}}^{n}\left(1 / \delta_{2 k+1, n}^{m}\right)-\sum_{k=n_{0}}^{n}\left(2 / \gamma_{2 k, n}^{m}\right) \\
& -\sum_{k=n_{0}}^{\infty}\left[\left(1 / \delta_{2 k}^{m}\right)+\left(1 / \delta_{2 k+1}^{m}\right)-2 / \gamma_{2 k}^{m}\right] \mid \\
& \leqq \\
& =\left(2 / \sup _{2 n_{0}}\right)+\left(1 / \delta_{2 n_{0}, n}^{m}\right)+\left(1 / \delta_{2 n_{0}+1, n}^{m}\right)+\left(1 / \delta_{2 n_{0}}^{m}\right)+\left(1 / \delta_{2 n_{0}+1}^{m}\right) .
\end{aligned}
$$

Since $\delta_{2 n_{0}} \rightarrow \infty$ and $\delta_{2 n_{0}+1} \rightarrow \infty$ as $n_{0} \rightarrow \infty$, it follows that the limit as $n \rightarrow \infty$ of each coefficient of the preceding expansion of $\log \left[R_{n}(z) / \pi(z)\right]$ is zero. Furthermore, because as $n \rightarrow \infty,\left\{\log \left[R_{n}(z) / \pi(z)\right]\right\}_{n=1}^{\infty}$ converges uniformly on $\{|z|<r\}$, then $\log \left[R_{n}(z) / \pi(z)\right] \rightarrow 0$ as $n \rightarrow \infty$. Thus $\pi(z)=\lim _{n \rightarrow \infty} R_{n}(z)=f(z)$.

LEMma 7. $\sum_{k=0}^{\infty} 1 / \alpha_{2 k+1}<\infty, \sum_{k=1}^{\infty} 1 / \beta_{k}<\infty, \sum_{k=1}^{\infty} 1 / \gamma_{2 k}<\infty$, and

$$
\sum_{k=2}^{\infty} 1 / \delta_{k}<\infty \text {. }
$$

Proof. Again by Lemma 4, there exists $r>0$ such that $f^{\prime}(z) \neq 0$ and $R_{n}^{\prime}(z) \neq 0$ for $|z|<r$. Since $R_{n}(z) \rightarrow f(z)$, it follows that $R_{n}^{\prime}(z) \rightarrow$ $f^{\prime}(z)$ and thus $\log R_{n}^{\prime}(z) \rightarrow \log f^{\prime}(z)$ uniformly in $\{|z|<r\}$ as $n \rightarrow \infty$. Thus for $|z|<r, \log R_{n}^{\prime}(z)$

$$
\begin{aligned}
= & \sum_{m=1}^{\infty}\left[z^{m} / m\right]\left[-\sum_{k=0}^{n} 1 / \alpha_{2 k+1, n}^{m}\right. \\
& \left.+\sum_{k=1}^{2 n+1}(-1)^{m+1} / \beta_{k, n}^{m}+2(-1)^{m} / \gamma_{1, n}^{m}+\sum_{k=1}^{n} 3 / \gamma_{2 k, n}^{m}\right] .
\end{aligned}
$$

Hence, for $m=1$,

$$
\lim _{n \rightarrow \infty}\left|-\sum_{k=0}^{n} 1 / \alpha_{2 k+1, n}+\sum_{k=1}^{2 n+1} 1 / \beta_{k, n}-2 / \gamma_{1, n}+\sum_{k=1}^{n} 3 / \gamma_{2 k, n}\right|<\infty .
$$

Because $0<\gamma_{1, n}<\beta_{1, n}$ and $0<\gamma_{2 k, n}<\alpha_{2 k+1, n}$, then

$$
\begin{aligned}
0 & <\sum_{k=1}^{2 n+1} 1 / \beta_{k, n}+\sum_{k=1}^{n} 2 / \gamma_{2 k, n} \\
& <-\sum_{k=0}^{n} 1 / \alpha_{2 k+1, n}+\sum_{k=1}^{2 n+1} 1 / \beta_{k, n}-2 / \gamma_{1, n} \\
& +\sum_{k=1}^{n} 3 / \gamma_{2 k, n}+1 / \alpha_{1, n}+2 / \gamma_{1, n} .
\end{aligned}
$$

Therefore, as $n \rightarrow \infty$,

$$
0 \leqq \lim \sup \left[\sum_{k=1}^{2 n+1} 1 / \beta_{k, n}+\sum_{k=1}^{n} 2 / \gamma_{2 k, n}\right]<\infty,
$$




$$
\lim \sup \sum_{k=1}^{2 n+1} 1 / \beta_{k, n}<\infty \text {, and } \lim \sup \sum_{k=1}^{n} 1 / \gamma_{2 k, n}<\infty .
$$

Furthermore, because for

$$
\begin{gathered}
k \geqq 1, \gamma_{2 k, n}<\delta_{2 k+1, n}<\alpha_{2 k+1, n}<\delta_{2 k+2, n}, \\
\lim _{n \rightarrow \infty} \sup _{\sum_{k=3}^{2 n+2}} 1 / \delta_{k, n} \leqq \lim _{n \rightarrow \infty} \sup _{k=1}^{n} 2 / \gamma_{2 k, n}<\infty
\end{gathered}
$$

and

$$
\lim _{n \rightarrow \infty} \sup _{k=1}^{n} 1 / \alpha_{2 k+1, n} \leqq \lim _{n \rightarrow \infty} \sup _{k=1} \sum_{k=1}^{n} 1 / \gamma_{2 k, n}<\infty
$$

Hence

$$
\lim _{n \rightarrow \infty} \sup _{k=0}^{n} 1 / \alpha_{2 k+1, n}<\infty \text { and } \quad \lim \sup _{n \rightarrow \infty} \sum_{k=2}^{2 n+2} 1 / \delta_{k, n}<\infty .
$$

For all $N>0$, as $n \rightarrow \infty$,

$$
\sum_{k=0}^{N} 1 / \alpha_{2 k+1}=\sum_{k=0}^{N} \lim 1 / \alpha_{2 k+1, n} \leqq \lim \sup \sum_{k=0}^{n} 1 / \alpha_{2 k+1, n}<\infty,
$$

and thus $\sum_{k=0}^{\infty} 1 / \alpha_{2 k+1}<\infty$. The convergence of the other series is established in a similar manner.

LEMMA 8. Each of the three infinite products in

$$
P(z)=\left[1 /\left(1+z / \gamma_{1}\right)^{2}\right]\left[\prod_{k=0}^{\infty} \alpha_{2 k+1}^{*} \prod_{k=1}^{\infty} \beta_{k}^{*} / \prod_{k=1}^{\infty}\left(\gamma_{2 k}^{*}\right)^{3}\right]
$$

converges uniformly on any compact subset of the z-plane.

Proof. This is a consequence of Lemma 7.

Lemma 9. $f^{\prime}(z)=[\exp (\delta z)][P(z)]$ where $\delta$ is real.

Proof. By Lemma 4, there exists $r>0$ such that for $|z|<r$, $R_{n}^{\prime}(z) \neq 0$ and $f^{\prime}(z) \neq 0$. For $m \geqq 1$, consider the coefficient of $z^{m} / m$ in the Taylor expansion of $\log \left[R_{n}^{\prime}(z) / P(z)\right]$ about $z=0$ for $|z|<r$. Because of Lemma 7 , there exists $M>0$ such that for all $n \geqq 1$,

$$
\sum_{k=1}^{n} 1 / \gamma_{2 k, n}<M \text { and } \sum_{k=1}^{\infty} 1 / \gamma_{2 k}<M \text {. }
$$

Then because of the ordering of the $\gamma_{k, n}$ and $\gamma_{k}$, for each $k<n$, $k / \gamma_{2 k, n}<M$ and $k / \gamma_{2 k}<M$. Thus for each $N>1$, as $n \rightarrow \infty$, 


$$
\begin{aligned}
& \lim \sup \left|\sum_{k=1}^{n} 1 / \gamma_{2 k, n}^{m}-\sum_{k=1}^{\infty} 1 / \gamma_{2 k}^{m}\right| \\
\leqq & \lim \sup \left|\sum_{k=N}^{n} 1 / \gamma_{2 k, n}^{m}-\sum_{k=N}^{\infty} 1 / \gamma_{2 k}^{m}\right| \leqq 2 M^{m} \sum_{k=N}^{\infty} 1 / k^{m},
\end{aligned}
$$

which implies for $m \geqq 2$, as $n \rightarrow \infty$

$$
\lim \left[\sum_{k=1}^{n} 1 / \gamma_{2 k, n}^{m}-\sum_{k=1}^{\infty} 1 / \gamma_{2 k}^{m}\right]=0 .
$$

Similarly, the other terms in the coefficient of $z^{m} / m$ have a limit of zero for $m \geqq 2$, and the coefficient of $z$ is real. Then as $n \rightarrow \infty$, $\log \left[R_{n}^{\prime}(z) / P(z)\right] \rightarrow \log \left[f^{\prime}(z) / P(z)\right]=\delta z$, and thus $f^{\prime}(z)=[\exp (\delta z)][P(z)]$.

LEMMA $10 . \delta=0$.

Proof. Because the factors of $P(z)$ are canonical products of genus zero with real zeros, for $\varepsilon>0$ and $0<\rho \leqq|\arg z| \leqq \pi-\rho, P(z)=$ $0[\exp (\varepsilon|z|)]$ and $1 / P(z)=0[\exp (\varepsilon|z|)]$. Then if arg $z$ satisfies the preceding conditions and $|z|$ is sufficiently large, then

$$
\exp [\delta \mathscr{R}(z)-\varepsilon|z|] \leqq\left|f^{\prime}(z)\right| \leqq \exp [\delta \mathscr{R}(z)+\varepsilon|z|] .
$$

Let $A_{1}=\{z \mid \pi / 4 \leqq \arg z \leqq \pi / 3\}$ and $A_{2}=\{z \mid 2 \pi / 3 \leqq \arg z \leqq 3 \pi / 4\}$. If $\delta>0$, then there exists $\varphi_{1}>0$ such that for $|z|$ sufficiently large $\left|f^{\prime}(z)\right| \geqq \exp \left(\varphi_{1}|z|\right)$ when $z \in A_{1}$ and $\left|f^{\prime}(z)\right| \leqq \exp \left(-\varphi_{1}|z|\right)$ when $z \in A_{2}$. Thus as $z \rightarrow \infty$ in $A_{2}, f^{\prime}(z) \rightarrow 0$, and because $f(z) \geqq b_{2 n}>0$ for $z$ on the curve $C_{2 n}, f(z) \rightarrow k \geqq 0$ as $z \rightarrow \infty$ in $A_{2}$. Thus for $n$ sufficiently large, $b_{2 n}<k+1$. Since $f^{\prime}(z) d z>0$ in the positive sense on the part of the curve $C_{2 n+1}$ in $A_{1}, b_{2 n+1}-a_{2 n+1} \rightarrow \infty$ as $n \rightarrow \infty$, where $a_{2 n+1}>0$ and thus $b_{2 n+1} \rightarrow \infty$ as $n \rightarrow \infty$. Because $b_{2 n+1}<b_{2 n}$, a contradiction has been reached and $\delta \ngtr 0$. If $\delta<0$, then there exists $\varphi_{2}>0$ such that for $|z|$ sufficiently large $\left|f^{\prime}(z)\right| \geqq \exp \left(\varphi_{2}|z|\right)$ when $z \in A_{2}$ and $\left|f^{\prime}(z)\right| \leqq \exp \left(-\varphi_{2}|z|\right)$ when $z \in A_{1}$. Similarly, $\delta \nless 0$ 。

THEOREM. A Riemann surface of the class defined is parabolic and its mapping function $f$ is given by

$$
f(z)=\left[z /\left(1+z / \gamma_{1}\right)\right] \prod_{k=1}^{\infty}\left[\delta_{2, k}^{*} \delta_{2, k+1}^{*} /\left(\gamma_{2 k}^{*}\right)^{2}\right]
$$

where

$$
f^{\prime}(z)=\left[1 /\left(1+z / \gamma_{1}\right)^{2}\right]\left[\prod_{k=0}^{\infty} \alpha_{2 k+1}^{*} \prod_{k=1}^{\infty} \beta_{k}^{*} / \prod_{k=1}^{\infty}\left(\gamma_{2 k}^{*}\right)^{3}\right] .
$$

Furthermore,

$$
\sum_{k=0}^{\infty} 1 / \alpha_{2 k+1}<\infty, \sum_{k=1}^{\infty} 1 / \beta_{k}<\infty, \sum_{k=1}^{\infty} 1 / \gamma_{2 k}<\infty, \text { and } \sum_{k=2}^{\infty} 1 / \delta_{k}<\infty .
$$


REMARKS. Lemmas 5 and 6 establish the representation of $f(z)$ as the product of quotients, while Lemmas 8 and 9 show a representation of $f^{\prime}(z)$ as a quotient of products. However, Lemma 7 can be used to show that the representation of $f(z)$ can also be considered as the quotient of products.

\section{REFERENCE}

1. G. R. MacLane, Riemann surfaces and asymptotic valies associated with real entire functions, The Rice Institute Pamphlet (1952).

The UNIVERSITY OF TEXAS 


\title{
PACIFIC JOURNAL OF MATHEMATICS
}

\author{
EDITORS
}

\author{
H. SAmelson \\ Stanford University \\ Stanford, California \\ R. M. Blumenthal \\ University of Washington \\ Seattle, Washington 98105
}

\author{
J. DugundjI \\ University of Southern California \\ Los Angeles, California 90007
}

*Richard Arens

University of California

Los Angeles, California 90024

\section{ASSOCIATE EDITORS}
E. F. BECKENBACH
B. H. NeUmanN
F. WOLF
K. YoSIDA

\section{SUPPORTING INSTITUTIONS}

\author{
UNIVERSITY OF BRITISH COLUMBIA \\ CALIFORNIA INSTITUTE OF TECHNOLOGY \\ UNIVERSITY OF CALIFORNIA \\ MONTANA STATE UNIVERSITY \\ UNIVERSITY OF NEVADA \\ NEW MEXICO STATE UNIVERSITY \\ OREGON STATE UNIVERSITY \\ UNIVERSITY OF OREGON \\ OSAKA UNIVERSITY \\ UNIVERSITY OF SOUTHERN CALIFORNIA
}

\author{
STANFORD UNIVERSITY \\ UNIVERSITY OF TOKYO \\ UNIVERSITY OF UTAH \\ WASHINGTON STATE UNIVERSITY \\ UNIVERSITY OF WASHINGTON \\ * * * * \\ AMERICAN MATHEMATICAL SOCIETY \\ CALIFORNIA RESEARCH CORPORATION \\ SPACE TECHNOLOGY LABORATORIES \\ NAVAL ORDNANCE TEST STATION
}

Mathematical papers intended for publication in the Pacific Journal of Mathematics should by typewritten (double spaced). The first paragraph or two must be capable of being used separately as a synopsis of the entire paper. It should not contain references to the bibliography. No separate author's resumé is required. Manuscripts may be sent to any one of the four editors. All other communications to the editors should be addressed to the managing editor, Richard Arens, at the University of California, Los Angeles, California 90024.

50 reprints per author of each article are furnished free of charge; additional copies may be obtained at cost in multiples of 50 .

The Pacific Journal of Mathematics is published quarterly, in March, June, September, and December. Effective with Volume 13 the price per volume (4 numbers) is $\$ 18.00$; single issues, $\$ 5.00$. Special price for current issues to individual faculty members of supporting institutions and to individual members of the American Mathematical Society: $\$ 8.00$ per volume; single issues $\$ 2.50$. Back numbers are available.

Subscriptions, orders for back numbers, and changes of address should be sent to Pacific Journal of Mathematics, 103 Highland Boulevard, Berkeley 8, California.

Printed at Kokusai Bunken Insatsusha (International Academic Printing Co., Ltd.), No. 6, 2-chome, Fujimi-cho, Chiyoda-ku, Tokyo, Japan.

PUBLISHED BY PACIFIC JOURNAL OF MATHEMATICS, A NON-PROFIT CORPORATION

The Supporting Institutions listed above contribute to the cost of publication of this Journal, but they are not owners or publishers and have no responsibility for its content or policies.

* Basil Gordon, Acting Managing Editor until February 1, 1966. 


\section{Pacific Journal of Mathematics}

\section{Vol. 15, No. $4 \quad$ December, 1965}

Robert James Blattner, Group extension representations and the structure space ........... 1101

Glen Eugene Bredon, On the continuous image of a singular chain complex .............. 1115

David Hilding Carlson, On real eigenvalues of complex matrices .................... 1119

Hsin Chu, Fixed points in a transformation group ............................. 1131

Howard Benton Curtis, Jr., The uniformizing function for certain simply connected Riemann

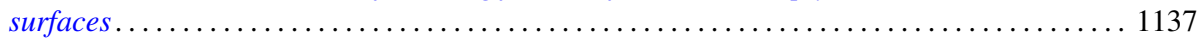

George Wesley Day, Free complete extensions of Boolean algebras................... 1145

Edward George Effros, The Borel space of von Neumann algebras on a separable Hilbert

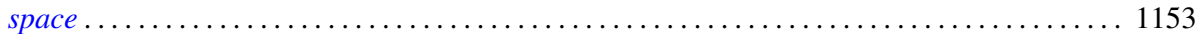

Michel Mendès France, $A$ set of nonnormal numbers ......................... 1165

Jack L. Goldberg, Polynomials orthogonal over a denumerable set ................ 1171

Frederick Paul Greenleaf, Norm decreasing homomorphisms of group algebras . . . . . . . . 1187

Fletcher Gross, The 2-length of a finite solvable group ........................ 1221

Kenneth Myron Hoffman and Arlan Bruce Ramsay, Algebras of bounded sequences ........ 1239

James Patrick Jans, Some aspects of torsion . . . . . . . . . . . . . . . . . . . . . . . 1249

Laura Ketchum Kodama, Boundary measures of analytic differentials and uniform

approximation on a Riemann surface ............................... 1261

Alan G. Konheim and Benjamin Weiss, Functions which operate on characteristic

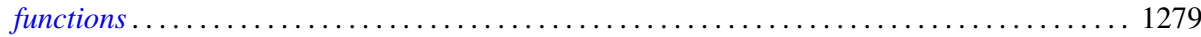

Ronald John Larsen, Almost invariant measures ............................ 1295

You-Feng Lin, Generalized character semigroups: The Schwarz decomposition ............ 1307

Justin Thomas Lloyd, Representations of lattice-ordered groups having a basis . . . . . . . . 1313

Thomas Graham McLaughlin, On relative coimmunity ....................... 1319

Mitsuru Nakai, $\Phi$-bounded harmonic functions and classification of Riemann surfaces ....... 1329

L. G. Novoa, On n-ordered sets and order completeness ..................... 1337

Fredos Papangelou, Some considerations on convergence in abelian lattice-groups . . . . . . . 1347

Frank Albert Raymond, Some remarks on the coefficients used in the theory of homology

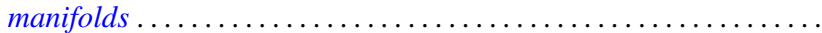

John R. Ringrose, On sub-algebras of a $C^{*}$-algebra .

Jack Max Robertson, Some topological properties of certain spaces of differentiable

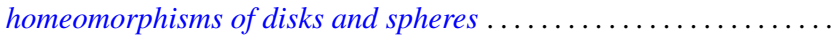

Zalman Rubinstein, Some results in the location of zeros of polynomials

Arthur Argyle Sagle, On simple algebras obtained from homogeneous general Lie triple systems. . . .

Hans Samelson, On small maps of manifolds ............................... 1401

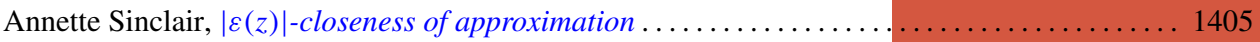

Edsel Ford Stiel, Isometric immersions of manifolds of nonnegative constant sectional curvature

Earl J. Taft, Invariant splitting in Jordan and alternative algebras ................. 1421

L. E. Ward, On a conjecture of R. J. Koch . . . . . . . . . . . . . . . . . . . . . . . . . . . 1429

Neil Marchand Wigley, Development of the mapping function at a corner . . . . . . . . . . 1435

Horace C. Wiser, Embedding a circle of trees in the plane ....................... 1463

Adil Mohamed Yaqub, Ring-logics and residue class rings . . . . . . . . . . . . . . . . 1465

John W. Lamperti and Patrick Colonel Suppes, Correction to: Chains of infinite order and their application to learning theory ........................................ 1471

Charles Vernon Coffman, Correction to: Non-linear differential equations on cones in Banach

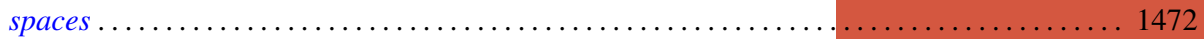

P. H. Doyle, III, Correction to: A sufficient condition that an $\operatorname{arc}$ in $S^{n}$ be cellular. . . . . . . . 1474

P. P. Saworotnow, Correction to: On continuity of multiplication in a complemented algebra 